\title{
Acyclovir resistant varicella zoster and HIV infection
}

E G H Lyall, M M Ogilvie, N M Smith, S Burns

\begin{abstract}
A child infected with HIV who developed chronic varicella zoster virus infection resistant to acyclovir is presented. The clinical course of the infection, treatment, virological investigations, and relationship of the infection to the child's immunodeficient state are discussed.

(Arch Dis Child 1994; 70: 133-135)
\end{abstract}

Varicella zoster virus can cause devastating disease in immunosuppressed patients after either primary or recurrent infection. The outcome of such infections improved dramatically when acyclovir became available. ${ }^{1}$ Chronic varicella zoster virus infection with superficial lesions lasting for months, sometimes disseminating at a later stage, has also been described in immunosuppressed patients ${ }^{2}$ and in some of these cases the virus has been resistant to acyclovir. $^{3}$

\section{Case history}

An infant contracted HIV from a blood transfusion at 0.3 years of age. The course of his illness from age 4 is demonstrated in fig 1 . While receiving intravenous gammaglobulin (IVIG) infusions at 6 years he had mild, untreated, varicella zoster infection. A year later treatment of progressive lymphoid interstitial pneumonitis with initially high dose then low dose maintenance prednisolone coincided with a sharp decline in CD4 count which never returned to presteroid levels. Seven months after starting steroids varicella zoster lesions affecting the distribution of the right trigeminal nerve (maxillary and mandibular divisions), were treated successfully with intravenous then oral acyclovir, which was continued long term with the intention of preventing recurrence. Soon after, the right eye became red and painful, and chronic varicella zoster virus keratitis was considered the most likely diagnosis. Topical treatment with acyclovir and steroids reduced but never completely eradicated the inflammation. Three months later he presented with life threatening respiratory failure and encephalopathy, subsequently attributed to parainfluenza virus type 3 which was isolated from throat and urine. Empirical treatment with intravenous acyclovir was started at the onset and after two days attenuated bilateral thoracic zoster developed.
Royal Hospital for Sick Children, Sciennes Road, Edinburgh EH9 1LF, Department of Haematology E G H Lyall

Department of Paediatric Pathology N M Smith

Department of Medical Microbiology, University of Edinburgh

M M Ogilvie

Regional Virus Laboratory, City Hospital, Edinburgh

$S$ Burns

Correspondence to: Dr Lyall.

Accepted 28 September 1993

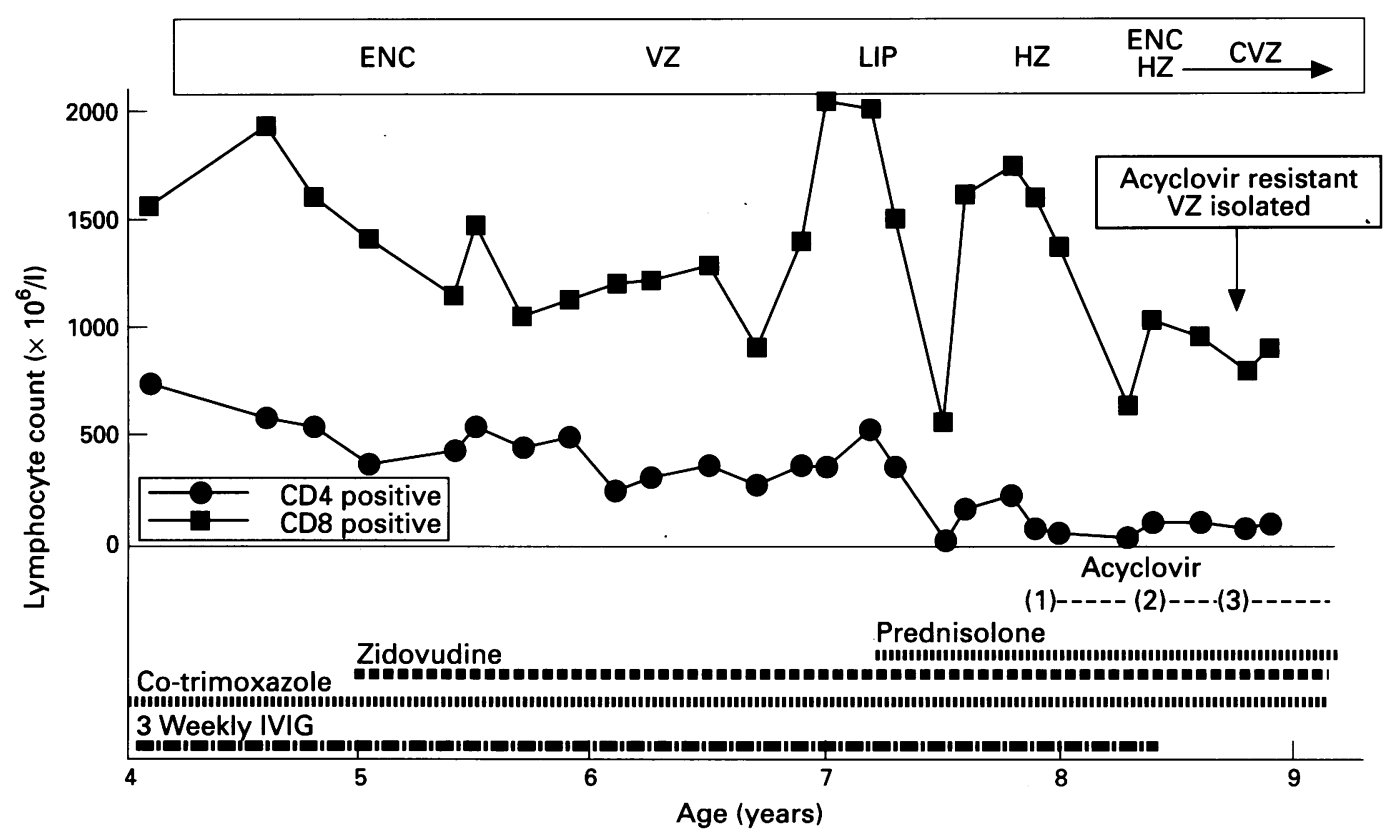

Figure 1 Sequential CD4 and CD8 counts, major clinical events and chemotherapy in patient studied. Upper box denotes illnesses: $E N C=$ encephalopathic illness; $V Z=$ primary varicella zoster; $L I P=$ severe deterioration in lymphoid interstitial pneumonitis; $H Z=$ herpes zoster; $C V Z=$ chronic varicella zoster. Lower lines denote significant medications. IVIG: $200 \mathrm{mg} / \mathrm{kg}$ every three weeks; co-trimoxazole: $120 \mathrm{mg}$ oral $\times 3$ per week; zidovudine: $370 \mathrm{mg} / \mathrm{m}^{2} /$ day for two years, then increased to $720 \mathrm{mg} / \mathrm{m}^{2} /$ day for 15 months, $600 \mathrm{mg} / \mathrm{m}^{2} /$ day for last nine months of life - always oral doses three times a day; prednisolone: $2 \mathrm{mg} / \mathrm{kg} /$ day for one month, reduced over two months and maintained at 5 mg alternate days.

Acyclovir: (1) intravenous, $10 \mathrm{mg} / \mathrm{kg} /$ dose, three times a day, $4-5$ days; then $800 \mathrm{mg}$ oral $\times 5$ per day for 5 days; followed by oral maintenance, $200 \mathrm{mg}$ four times a day. (2) Intravenous, $10 \mathrm{mg} / \mathrm{kg} /$ dose, three times a day, 9 days; followed by oral maintenance, $200 \mathrm{mg}$ four times a day. (3) Intravenous, $10 \mathrm{mg} / \mathrm{kg} /$ dose, three times a day, 10 days; followed by oral maintenance, $400 \mathrm{mg}$ four times a day. 


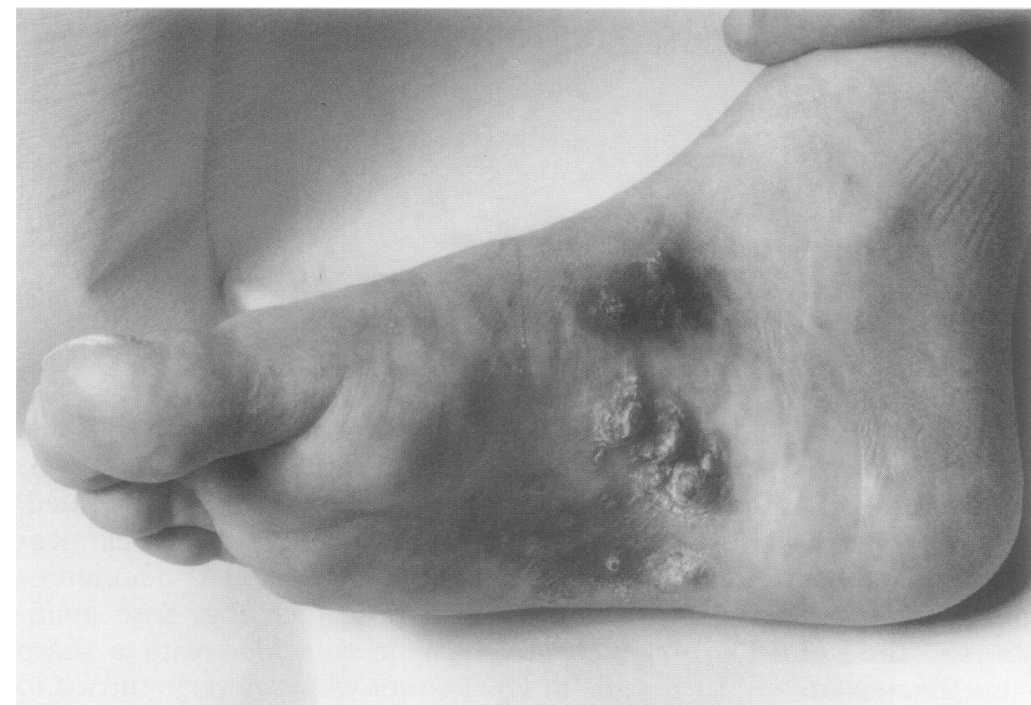

Figure 2 Sole of the right foot: heavily keratinised older varicella zoster lesions surrounded by newer satellite lesions. to acyclovir was confirmed at the Wellcome Research Laboratories in a plaque reduction assay. The isolate had an acyclovir $50 \%$ inhibitory concentration $\left(\mathrm{IC}_{50}\right)$ of $709 \mu \mathrm{M}$, when the sensitive/resistant threshold $\mathrm{IC}_{50}$ was $30 \mu \mathrm{M}$. The virus was sensitive to phosphonacetic acid, the parent compound of foscarnet. Histology of the biopsied plantar lesion was consistent with varicella zoster virus infection (fig 3). Serological response to varicella zoster virus was unhelpful as the patient was receiving IVIG.

\section{Discussion}

Chronic varicella lesions similar to our patient's were reported from immunosuppressed patients in 1979 before the advent of acyclovir or HIV (two transplant patients and two with lymphoproliferative diseases). ${ }^{2}$ In these cases when immunosuppressive treatment was reduced the lesions healed spontaneously. Whether hyperkeratotic chronic infection is associated with acyclovir resistant strains or related to inadequate host response remains unknown. Corticosteriods depress cellular immunity, and such treatment combined with HIV related immunodeficiency in our patient severely suppressed the response to varicella zoster virus allowing reactivation of virus. The subsequent increased viral load in the presence of acyclovir could have increased the chance of acyclovir resistant mutant strains emerging. Indeed, suboptimal acyclovir blood concentrations have been demonstrated in HIV patients Within one month new painful spots began to appear on the feet, trunk, neck, legs, arms and face, with deterioration in respiratory function and vision without evidence of other infection. Foscarnet $(200 \mathrm{mg} / \mathrm{kg} /$ day continuous intravenous infusion) was introduced but only tolerated for six days. Despite the inadequate treatment the lesions stopped cropping and some of the long accumulated crusts detached, revealing healed skin beneath. Subsequent deterioration in the child's condition led to withdrawal of all antiviral treatment, and within 3-4 weeks painful varicella lesions were widely disseminated. Encephalopathy supervened and the child died nine years after contracting HIV. Necropsy was not performed.

\section{Methods and results}

Despite multiple sampling from vesicular lesions, the eye and the plantar lesions, there was evidence of varicella zoster virus antigen by immunofluorescence on only one occasion in the last stage before commencing foscarnet. All routine cultures for varicella zoster virus were negative, and cultures for other viruses, bacteria, fungi, and mycobacteria were repeatedly negative. The plantar lesions were aspirated and swabbed twice and biopsied once, but there was no vesicular fluid for electron microscopy. The single varicella zoster virus isolate was obtained from a swab of a plantar lesion after prolonged cell culture in a 'shell vial' system and was found to be resistant to acyclovir at concentration of $100 \mu \mathrm{M}$ in this system by MMO. Resistance

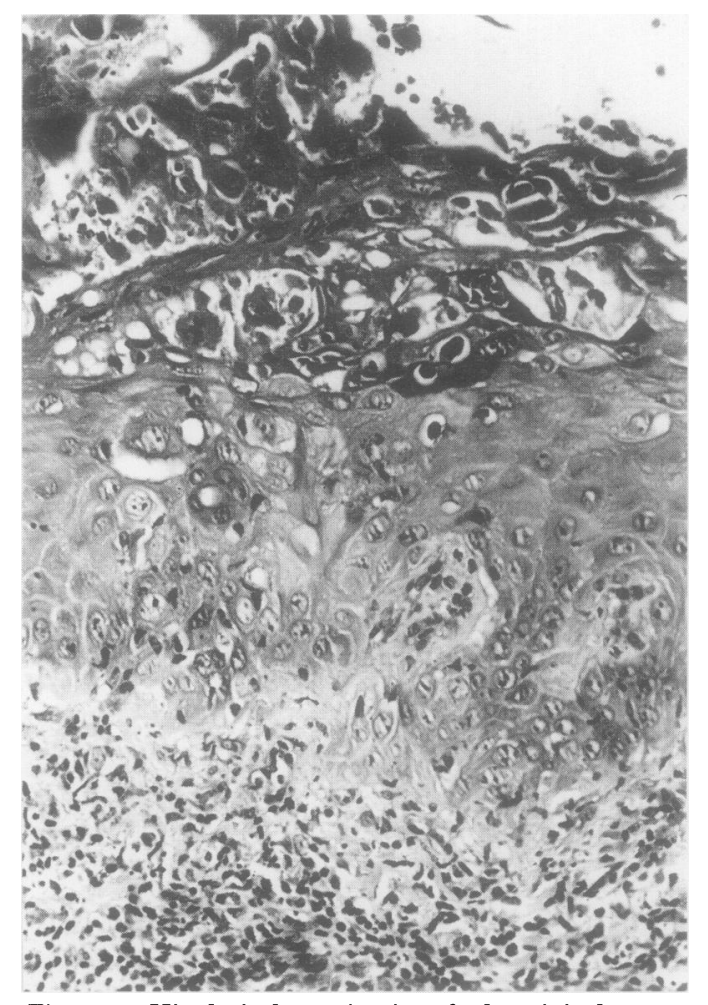

Figure 3 Histological examination of a keratinised plantar lesion: there is superficial dermal lymphohistiocytic infiltrate approaching the basal epidermis. The epidermal layer is abnormal, with multinucleate cells and many keratinocytes are larger than normal with large nuclei and prominent nucleoli. An intraepidermal vesicle lined by degenerate cells is present. (Haematoxylin and eosin, original magnification $\times 250$.) 
on long term oral treatment who developed chronic lesions of varicella zoster virus. ${ }^{4}$

Fifteen cases of chronic varicella zoster virus infection have been described in patients infected with HIV and all had received acyclovir. ${ }^{5}$ Our isolate appears to be the first reported in the UK and shares important similarities with the first case of acyclovir resistant varicella zoster virus described by Pahwa et al in a 4 year old girl with AIDS $^{3}$ : primary varicella occurred while on IVIG treatment; initial attacks of dermatomal zoster healed with intravenous acyclovir and chronic lesions appeared later. Continuous oral treatment and multiple courses of intravenous acyclovir resulted in modest or no improvement in these lesions; varicella zoster virus was only isolated after eight months of acyclovir treatment (nine months in our case) and this was the isolate found to be acyclovir resistant. Unfortunately no pretreatment isolate was available from either child.

As acyclovir resistant varicella zoster virus remains rare, it is important to obtain optimal diagnostic samples from all eruptions in immunosuppressed patients before treatment and any isolates should be stored to test sensitivity to acyclovir if necessary. Treatment of varicella zoster virus should be with appropriate intravenous doses of acyclovir (in children calculated per $\mathrm{m}^{2}$ ) for at least 10 days and, where possible, additional immunosuppression should be avoided. The few acyclovir resistant cases treated for visceral dissemination have responded to foscarnet, which had a temporary effect in our patient. ${ }^{6}$ Clinicians should be aware of the atypical varicella lesions that can occur in immunosuppressed patients and that acyclovir resistant viral strains can emerge in these cases.

With thanks to $\mathrm{Mr} M$ Trowbridge, Wellcome Research Laboratories, who carried out the plaque reduction assay on this isolate of varicella zoster virus and to $\mathrm{Mr} \mathrm{L}$ Cummings, Royal Hospital for Sick Children, for photography.

1 Feldman S, Lott L. Varicella in children with cancer: impact of antiviral therapy and prophylaxis. Pediatrics 1987; 80 465-72.

2 Gallagher JG, Merigan TC. Prolonged herpes zoster infection associated with immunosuppressive therapy. Ann Intern Med 1979; 91: 842-6.

3 Pahwa S, Biron K, Lim W, et al. Continuous varicella zoster Pahwa S, Biron K, Lim W, et al. Continuous varicella zoster infection associated with acyclovir resis

4 Jacobson MA, Berger TG, Fikrig S, et al. Acyclovir resistant varicella zoster infection after chronic oral acyclovir therapy in patients with the acquired immunodeficiency syndrome. Ann Intern Med 1990; 112: 187-91.

5 Leibovitz E, Kaul A, Rigaud M, Bebenroth D, Krasinski K Borkowsky W. Chronic varicella zoster in a child infected with human immunodeficiency virus: case report and review of the literature. Cutis 1992; 49: 27-31.

6 Hoppenjans WB, Bibler MR, Orme RL, Solinger AM. Prolonged cutaneous herpes zoster in acquired immunodeficiency. Arch Dermatol 1990; 126: 1048-50. 